\title{
The intricacy of memory span
}

\author{
MICHAEL J. WATKINS \\ Princeton University, Princeton, New Jersey 08540
}

\begin{abstract}
The effects of word frequency on memory span were explored using the "up-and-down" method. Mean spans were greatest when the sequences were of all high-frequency words (5.82), and smallest when they were of all low-frequency words (4.24). For mixed-frequency sequences, mean spans were greater when the high-frequency words were presented before the low-frequency words (5.19) than when the low-frequency words came first (4.65). The findings are discussed in terms of the primary-secondary memory distinction worked out for single-trial free recall, and the logic of this distinction is used to argue against attributing span performance to a simple unitary process.
\end{abstract}

The concept of memory span is as old as the experimental study of memory itself. Thus, in the very first such investigation, Ebbinghaus (1885/1964) found that he could almost always reproduce a series of up to seven items after just a single presentation, whereas for longer series correct reproduction usually required many presentations. Shortly after publication of Ebbinghaus' monograph, it was shown that the immediate memory span, or "span of prehension," was a valid measure of mental capacity (Galton, 1887; Jacobs, 1887), and it soon found its place in clinical diagnosis and intelligence testing.

Despite its splendid history, the concept of memory span has been subjected to remarkably little critical analysis. Today we know hardly any more about memory span, about the extraordinary effect of increasing list length over a comparatively short range, than Ebbinghaus knew. This neglect is made the more striking by the intensive current interest in the immediate serial recall of supraspan lists. No doubt the primary reason for this discrepancy lies in the difference in the readiness with which span and supraspan recall submit to analysis. In particular, the items of supraspan lists can be classified according to whether or not they are recalled, thereby allowing serial position functions to be drawn, analyzed, and given a theoretical interpretation. With span recall, on the other hand, we lack suitable techniques for revealing any influence of serial position. The purpose of this paper is to suggest one such technique, and to use it to test the hypothesis that span recall is the product of a simple unitary process.

Before proceeding to the experiment, it is perhaps important to consider the relevance for span recall of the findings and theories of supraspan research. It might be held that whether list length is just short

This research was supported by National Research Council of Canada Grant A8632 to Endel Tulving. Requests for reprints may be sent to Michael $J$. Watkins at the Department of Psychology, Princeton University, Princeton, New Jersey 08540. enough or just too long for perfect reproduction has little effect on the underlying mechanisms. We will argue that there are currently few if any good reasons for this view. A rather different view is that, unlike supraspan recall, the perfect reproduction of a list reflects a basic attribute of mental functioning, a fundamental property about which there is little to say. A further section of this paper considers and rejects a specific version of this notion, namely, that span is the product of primary memory. The experiment will then be described and we will see that its results are contrary to the general version of the latter view, and suggest rather that the mechanism underlying span recall is complex.

\section{What is Memory Span?}

Before considering the relation of memory span to supraspan recall and to primary memory, it is important to be quite clear about what we mean by memory span. Because of an inevitable variation in a person's performance, memory span is a statistical concept: It is the length of a series for which probability of immediate reproduction following a single presentation is at some arbitrary level between 0.0 and 1.0. With rare exceptions, the critical probability has been set at .5 , and thus for all practical purposes span is defined as the length of a list for which there is an even chance of perfect recall.

It should be noted that the term memory span is sometimes used in a much looser sense. Thus, it may be applied to a variety of immediate supraspan recall procedures, as well as to various indices of performance. Such extended usage is usually inappropriate. For instance, to say that memory span varies with the number of list items (Baddeley, Thomson, \& Buchanan, 1975 ) is analogous to saying that the volume of a glass varies with the amount of liquid it can contain.

Although it is necessary to vary list length in order to measure span, it is permissible to make an ordinal comparison of spans using data obtained with a fixed list-length procedure-collected, perhaps, for the primary 
purpose of constructing serial position functions. Thus, if the proportions of lists recalled without error differ significantly across conditions, then, given certain not unreasonable assumptions, it may be concluded that the memory spans corresponding to these conditions of presentation, type of material, and so on, are ordinally related to each other in the same way. However, fixed list-length data do not allow estimates of absolute values of span, or of the differences between them.

\section{Span Recall and Supraspan Recall}

There can be little doubt that any complete account of recall performance observed with supraspan lists will entail a complex process, or more likely a complex of several processes. The very fact that some items are recalled and others are not suggests a certain complexity in underlying processes. Moreover, after the first list or two the subject will realize that he is unlikely to manage perfect recall, and so he will presumably adopt some strategy or other for maximizing his performance. If it is assumed that such strategies affect the level, or at least the pattern, of recall, then presumably they should be reflected in any adequate theoretical account of performance.

There are also empirical reasons for supposing supraspan recall to be a complex phenomenon. In the first place, probability of recall is related to serial position. Moreover, this relation is not a simple monotonic one. Second, and perhaps more important, certain variables, including item similarity (Watkins, Watkins, \& Crowder, 1974), the frequency of item usage (Watkins \& Watkins, in press), the subject's overall recall ability (Murdock, 1968), and knowledge of list length (Crowder, 1969), interact with serial position; such interactions require relatively sophisticated interpretations.

There are, then, good reasons for not attributing supraspan serial recall to a simple unitary process. But the important point for the present purposes is that none of these reasons can be validly extended to the errorless performance of span recall. To argue that the minor change from the span to the supraspan procedure is a small price to pay for the power of serial position analysis could be to miss an important point, since it is possible that the very breakdown of recall in supraspan lists signals the use of relatively complex processes that are not involved when lists are short enough for perfect recall. Introspectively, there is nothing complicated in repeating a subspan list, such as a pair of digits. It would seem, then, that somewhere between a two-item list and say a supraspan, nine-item list, the recall process becomes complex. This complexity may build up continuously as list length is increased, or it may occur suddenly at some critical point. If the former is true then presumably our instrospections are in error, for the memory process involved in repeating a pair of digits will be in some degree complex. If the latter is true, then the length corresponding to span would seem an obvious possibility for a discontinuity of process.

\section{Memory Span and Primary Memory}

The term primary memory has acquired several meanings, but for the present purposes it will be defined as the mechanism, or at least the principal mechanism, for recalling items from the last seven or so list positions in a single-trial free recall task; the recall of items from earlier list positions is attributed to secondary memory. This distinction reflects the results of a great deal of free recall research exploring the effects of many different experimental variables as a function of serial position. For instance, while overall level of recall is sensitive to such variables as presentation rate, list length, item familiarity, item similarity, and so on, this sensitivity is essentially confined to the prerecency serial positions. Conversely, the interpolation of a brief distractor task between presentation and test reduces very sharply recall of recency items, but has very little effect on recall of prerecency items. These and similar findings (for reviews see, e.g., Crowder, 1976; Glanzer, 1972) have most often been interpreted as the result of two memory systems or processes, one highly vulnerable to distraction but otherwise capable of supporting a strictly limited number of recently occurring items, the other more durable and without strict capacity limitations.

The concept of a distinct memory system dealing with the most recently attended events naturally suggests itself as the mechanism underlying span recall. Further, primary memory is often characterized as a simple buffer system from which information can be retrieved without difficulty, and so in this respect it is consistent with the notion that span recall is a product of a very basic process. There are, however, a number of problems with this hypothesis.

The first difficulty concerns primary memory capacity. Although the free recall recency effect typically spans about seven serial positions, the capacity of primary memory corresponds roughly to the average number of recency items recalled by a single subject from a single list, and this is typically about three items. Since this value is rather less than memory span, it seems that primary memory is too limited in its capacity to be the sole source of recall in the memory span task.

This difficulty can, however, be met with the argument that the area under the recency effect may underestimate the capacity of primary memory. There are two possibilities here. First, subjects may use primary memory in the free recall task to recall one or two prerecency items, as well as recency items. Second, the serial order of recall in the span task could yield a more efficient use of primary memory than occurs in free recall. If a given item is likely to be lost from primary memory as a result of item recall as well as subsequent item presentation, then, given certain assumptions, the use of primary memory could be 
maximized by recalling the items in their presentation order. In short, it seems that the discrepancy between memory span and the area under the free recall recency effect is not necessarily inconsistent with the assumption that memory span is wholly the product of primary memory.

There is, however, a more serious difficulty for the view that memory span is a purely primary memory phenomenon. Primary memory, as we are using the term here, has a capacity that is essentially independent of type of item (Sumby, 1963; Tulving \& Colotla, 1970). Consequently, if memory span is attributable entirely to primary memory, then it, too, should be independent of type of item. But there is evidence to the contrary. Crannel and Parrish (1957), for instance, found that memory span for digits was about $60 \%$ greater than that for random words. A variation of memory span with class of item is also apparent from reviews of the memory span literature (e.g., Blankenship, 1938; Brener, 1940; Cavanagh, 1972).

One possible way in which the stability of free recall recency could be reconciled with the variability of memory span would be to appeal to the difference in the range of items used in the two paradigms. The brevity of the span list and the instruction for ordered recall have allowed the use of small sets of items, such as digits and geometric shapes. The free recall procedure, on the other hand, requires a relatively large set of items, so that item variation is typically restricted to switching between various word classes. Unfortunately for this argument, there is evidence that even with the same variation in material, memory span shows an effect of type of item, whereas free recall recency does not. Specifically, memory span is substantially greater with phonologically dissimilar than phonologically similar words (Baddeley, 1966), while the free recall recency effect appears to be unaffected by this variable (Watkins et al., 1974).

A final difficulty for the view that memory span relies entirely on primary memory derives from the finding by Craik (1971) that memory span correlated across subjects more highly with secondary memory performance $(r=.72)$ than with primary memory performance $(r=.49)$, where primary and secondary memory measures were obtained with a free recall procedure. This finding suggests that memory span does not derive entirely from primary memory.

\section{Serial Position Effects in Memory Span?}

It seems, then, that there are serious difficulties in assigning span recall exclusively to primary memory. Unfortunately, this negative conclusion leaves unanswered our primary question: Is span the product of a simple unitary process? Thus it might be held that there are no retrieval problems for secondary memory when lists are of span length or less, and that span recall derives entirely from secondary memory; or that the distinction between primary and secondary memory (as adopted here) does not generalize to the span procedure, but that span is the product of some other simple process. On the other hand, it could be argued that span recall is a composite of two (or more) processes, and that the relative contribution of these processes in the recall of each item varies with its serial position. This possibility has, in fact, been raised by Craik (1968). Having found the capacity of primary memory to be rather less than span, Craik wrote: "It may be speculatively suggested that word-span responses are essentially a read-out of PM [primary memory] content but that this component is augmented by one or two words retrieved from SM [secondary memory] . The span-measurement situation emphasizes remembering the beginning of the list, so these first words may be rehearsed and thus retained in PM at the expense of later items. At recall the first words are read out from PM and occasionally the remaining one or two words may be retrieved by the search process [i.e., from SM] and added to the end of the string" (p. 1003).

The tentative tone of Craik's proposal betrays the absence of evidence relevant to the general question of the nature of span recall. The experiment to be described here was designed to help remedy this neglect. The strategy was one of exploring the effect of a variable as a function of serial position. If a variable has a greater effect at some serial positions than at others, then we can conclude that, in some sense, recall is the product of the composite of at least two processes whose relative importance varies according to serial position. This is, of course, precisely the logic behind the distinction between primary and secondary memory in immediate free recall.

There is, however, a big difference in the subtlety of the analyses that are possible for the free recall and span procedures. The dependent variable used in the free recall research is typically the probability of item recall, determined separately for each serial position. Thus, in the case of free recall, it is a very simple matter to decide whether the effects of, say, class of item or condition of presentation interact with those of serial position. But there can be no such analysis with span recall, for here the concern is with errorless performance. We therefore face the problem of comparing the effects of a variable at different serial positions without the aid of serial position functions.

The solution to this problem entailed manipulating the chosen variable within lists rather than between lists. To achieve maximum simplicity, only two levels of list position were considered: first half and second half. If span recall involves a unitary process, then the chosen variable should have equal effects on the two list halves, and therefore span should be independent of how the levels of this variable are allocated to list half. But if span is observed to change with the ordering of the levels of a variable, then we may conclude that a more complex interpretation is required, one acknowledging that the process of recalling an item varies with list position.

The variable investigated in the present experiment 
was that of item frequency. One reason for choosing item frequency is that it is one of the variables which interact with serial position in the single-trial free recall procedure: For prerecency positions common items are better recalled than rare items, whereas for recency positions frequency has little effect (Raymond, 1969; Sumby, 1963). In other words, item frequency is regarded as affecting secondary memory but not primary memory. The logic of this distinction is applied to the present experiment by comparing spans for two types of mixed-frequency lists, one in which the items for the first half of the list are common words and those of the second half rare, the other in which rare words occur in the first half of the list and common words in the second. A significant difference in span for these two types of lists would indicate that span is a relatively complex phenomenon. If, as Craik (1968) speculated, the first few items in span recall derive from primary memory and the last ones derive, at least in part, from secondary memory, then span should be higher when the infrequent words precede the frequent words, since in this condition primary memory (which is assumed equally effective for high- and low-frequency words) can be used for low-frequency words, leaving secondary memory for the high-frequency words, that is, for those items for which it is relatively efficient. Conversely, primary memory could be more important for recency than for primacy items, just as it is in free recall. In this case span should be greater when the high-frequency words appear before the low-frequency words.

In addition to the mixed-frequency lists, the experiment included lists in which all words were of high frequency, and lists in which all words were of low frequency, so that, in all, span was measured for four types of lists. The single-frequency lists should provide additional evidence on the nature of span recall. In particular, any difference between the spans for the two pure-frequency lists would presumably mark an upper limit for any difference between the spans for the mixed-frequency lists, so that a comparison of the two differences should provide an index of the extent to which the effect of frequency is localized in one of the list halves.

\section{METHOD}

\begin{abstract}
Materials
Two pools of 200 one-syllable words were formed from the Thorndike-Lorge word count such that the words of one pool were of high frequency (more than 100 per million) and those of the other were of low frequency (3 per million or less). Word lengths were identically distributed for the two pools. Each word was printed in capital letters on a small flash card.
\end{abstract}

\section{Subjects}

Twenty-four University of Toronto undergraduates participated for pay.

\section{Design and Procedure}

All subjects were presented with a sequence of 48 lists for immediate recall in serial order. The $\mathbf{4 8}$ lists comprised four conditions of 12 lists each. For HH lists all words were of high frequency; for LL lists all were of low frequency; for $\mathrm{HL}$ lists words in the first half of the list were frequent and those in the second half infrequent; and for LH lists the first-half words were infrequent and the second-half words frequent. When an HL or LH list involved an odd number of words, the first part of the list was made one word longer than the second for half of the subjects, and one word shorter for the other half of the subjects.

The object of the experiment was to estimate memory span for each type of list. This was achieved with the up-and-down method. Thus, the number of words in a list of a given condition was one greater than the last list in that condition if the latter had been recalled correctly, and one less if it had not been recalled correctly. With this method subjects stabilize to a success rate of about 50\%. Each successive block of four lists included one from each condition, with a separate random determination of the order of conditions being made for each subject-block. For the first block, all four lists were four words long. For the second block, list length for a given condition was three or five words, depending on performance for that condition in the first block; and so on for successive blocks.

The two word pools were shuffled for each subject. The experimenter constructed each list by taking the required number of cards from the appropriate pile(s). The cards were presented to the subject at a 1-sec rate with a blank card covering the last list card. On seeing the blank card, the subject began writing down the words in serial order, using a fresh sheet for each list. The subject was fully aware that credit was gained only if all items were recalled in a temporal order that matched exactly the presentation order. No time limit was imposed on recall, and the next trial began after a short break during which the experimenter prepared the next list.

\section{RESULTS AND DISCUSSION}

Since the up-and-down method constantly adjusts list length to keep the subject's rate for perfect reproduction at about the $50 \%$ level, memory span is estimated by the mean length of the presented lists. In making this estimate, the first few lists are best ignored, since they will reflect the arbitrary length of the first list. In the present case, each subject's span for each condition was estimated by averaging the list length for Lists 5-13. (Although only 12 lists were presented, the length of List 13 was given by the length and outcome of List 12.) The mean span values for each of the four conditions are shown in Table 1.

The most important result is the difference in span for the HL and LH lists. This difference was significant $[\mathrm{t}(23)=4.95, \mathrm{p}<.001]$, and it indicates that the two list halves were differentially affected by item frequency. In terms of the primary-secondary memory distinction, it implies that the relative contributions of primary and secondary memory varied across list half. Since span was greater when high-frequency words were presented early in the list, and bearing in mind the assumptions that secondary memory is more efficient for high-frequency words and that primary memory is relatively independent of frequency, it follows that secondary memory contributed more to the recall of early than of late items. Or, put another way, since memory span was higher when low-frequency items were presented in the second half of the list, the contribution 
Table 1

Mean Memory Span for High-Frequency (HH), Low-Frequency (LL), and Mixed-Frequency (HL and LH) Word Lists

\begin{tabular}{cccc} 
HH & HL & LH & LL \\
\hline 5.82 & 5.19 & 4.65 & 4.24 \\
\hline
\end{tabular}

of primary memory was greater for the second half of the list than the first.

A second point to note from Table 1 is that though frequency has a stronger effect for first-half words, it nonetheless has some effect for second-half words. Thus, mean span was significantly greater when the second-half words were common (the $\mathrm{HH}$ and $\mathrm{LH}$ lists) than rare (the HL and LL lists) $[\mathrm{t}(23)=5.73$, $p<.001]$. If we were to interpret this result in terms of the primary-secondary memory distinction, we would conclude that though secondary memory is relatively more important for the first half of the list, it is nevertheless used to some extent for second-half items. It would not follow, however, that secondary memory is operative over all serial positions. It would remain possible that recall of the last one or two items was entirely attributable to primary memory, but that this was not revealed in the experiment because the list-half analysis was too crude.

\section{CONCLUSIONS}

The essential conclusion to be drawn from these experimental findings is that memory span is the product of something more than a simple unitary process.

The finding that span for high-frequency words is greater than that for low-frequency words is merely a further demonstration of the variation of span according to class of item (cf. Crannel \& Parrish, 1957). Such a finding does not, of itself, tell us very much about the complexity of the span process. It could, for instance, be interpreted in terms of a simple limited capacity system with a rate of capacity consumption that varies with the nature of the item.

More important than the main effect of frequency is the interactive effect between frequency and serial position. Such a finding requires a relatively sophisticated interpretation, one subtle enough to allow an attribute to be more important at some serial positions than at others. One obvious theoretical approach is to postulate two processes or memory systems differing in their sensitivity to item frequency and in the serial positions at which they are most effective.

An interpretation of this sort has, of course, been proposed for single-trial free recall. Recall of items from the last few presentation positions is attributed to primary memory, recall of earlier items to secondary memory. Item frequency is one of the many variables that can have a substantial effect on secondary memory, but little if any effect on primary memory. It is therefore tempting to assume that these same two processes are involved in span recall, with primary memory relatively more important for recency items, just as with free recall. Although this hypothesis may seem reasonable, it should be regarded with caution. The generality of the distinction between primary and secondary memory beyond the single-trial free recall procedure is not well understood. In particular, it is even unclear whether the distinction can be validly applied to the supraspan serial recall procedure, for though with this procedure several variables have been shown to interact with serial position (see e.g., Crowder, 1969; Murdock, 1968; Watkins et al., 1974; Watkins \& Watkins, in press), the manner in which they do so appears to be less consistent than with free recall. And whatever doubt there is about the usefulness of the primary-secondary memory distinction for supraspan recall, there is at least as much doubt about its usefulness for span recall. However, for the present purposes the question is not critical. What is critical is the logic underlying the primary-secondary memory distinction, and not the validity or usefulness of the distinction itself. While further research showing whether the pattern of results obtained here by varying frequency can also be obtained by varying other attributes may prove useful in evaluating a two-process interpretation, the present results would seem to be sufficient for us to conclude that span recall requires an interpretation at least as complex as a two-process account.

Finally, two subsidiary points. First, the data reported here are consistent with Craik's $(1968,1971)$ proposal that memory span is the joint product of primary and secondary memory, and that the relative contribution of these two components varies with serial position. However, they run counter to his speculation that primary memory is relatively more important for items presented early in the list.

Second, if the primary-secondary memory distinction is to be applied to span recall, then the implication in the present data that the primary memory contribution to recall is greater for late (recency) than for early (primacy) items is contrary to the assumption sometimes made (e.g., Tulving \& Colotla, 1970; Waugh \& Norman, 1968) that a primary memory item is equally vulnerable to the presentation and to the recall of other list items, since it follows from this assumption that the relative contributions of primary and secondary memory in the memory span situation will be independent of list position. That the contribution of primary memory is greater for recency than for primacy positions implies that primary memory retention is less vulnerable to the recall of an item than it is to the presentation of an item. 


\section{REFERENCES}

BADDELEY, A. D. Short-term memory for word sequences as a function of acoustic, semantic, and formal similarity. Quarterly Joumal of Experimental Psychology, 1966, 18. 362-365.

Baddeley, A. D., Thomson, N., \& Buchanan, M. Word length and the structure of short-term mernory. Journal of Verbal Learning and Verbal Behavior, 1975, 14, 575-589.

Blankenship. A. B. Memory span: A review of the literature. Psychological Bulletin, 1938, 35, 1-25.

BRENER, R. An experimental investigation of memory span. Journal of Experimental Psychology, 1940, 26, 467-482.

Cavanagh, J. P. Relation between the immediate memory span and the memory search rate. Psychological Review, 1972, 79, 525.530.

CraIK, F. I. M. Two components in free recall. Journal of Verbal Learning and Verbal Behavior, 1968, 7, 996-1004.

CraIK, F. I. M. Primary memory. British Medical Bulletin, 1971. 27, 232-236.

Crannel, C. W., \& Parrish, J. M. A comparison of immediate memory span for digits, letters, and words. Journal of Psychology, 1957, 44, 319-327.

Crowder, R. G. Behavioral strategies in immediate memory. Journal of Verbal Learning and Verbal Behavior, $1969,8,524-528$.

CRowder, R. G. Principles of learning and memory. Hillsdale. N.J: Lawrence Erlbaum, 1976.

Ebinghaus, H. Memory: A contribution to experimental psychology. New York: Dover, 1964. (Originally published, 1885.)

Galton, F. Supplementary notes on "prehension" in idiots. Mind, $1887,12,79-82$.
Glanzer, M. Storage mechanisms in recall. In G. H. Bower (Ed.), The psychology of learning and motivation: Advances in research and theory (Vol. 5). New York: Academic Press, 1972.

JACOBS, J. Experiments on "prehension." Mind, 1887, 12, 75-79.

Murdock, B. B., JR. Serial order effects in short-term memory. Journal of Experimental Psychology Monograph Supplement, 1968, 76. (Part 2) 1-15.

RAYMOND, B. Short-term storage and long-term storage in free recall. Journal of Verbal Learning and Verbal Behavior, 1969, 8, 567-574.

SumbY, W. H. Word frequency and the serial position effect. Journal of Verbal Learning and Verbal Behavior, 1963, 1 , 443-450.

Tulving, E., \& Colotla, V. A. Free recall of trilingual lists. Cognitive Psychology, 1970, 1, 86-98.

Watkins, M. J., Watkins, O. C., \& Crowder, R. G. The modality effect in free and serial recall as a function of phonological similarity. Journal of Verbal Learning and Verbal Behavior, 1974, 13, 430-447.

Watkins, O. C., \& Watkins, M. J. On serial recall and the modality effect: Effects of word frequency. Journal of Experimental Psychology: Human Learning and Memory, in press.

Waugh, N. C., \& Norman, D. A. The measure of interference in primary memory. Joumal of Verbal Learning and Verbal Behavior, 1968, 7, 617-626.

(Received for publication April 15, 1977; revision accepted June $10,1977$. ) 